LA-UR-00- 385

Approved for public release;

distribution is unlimited

\title{
Title: $\quad$ Imaging Time-of-Flight Ion Mass spectrograph
}



\section{Los Alamos}

Los Alamos National Laboratory, an affirmative action/equal opportunity employer, is operated by the University of California for the U.S. Department of Energy under contract W-7405-ENG-36. By acceptance of this article, the publisher recognizes that the U.S. Government retains a nonexclusive, royaltyfree license to publish or reproduce the published form of this contribution, or to allow others to do so, for U.S. Government purposes. Los Alamos National Laboratory requests that the publisher identify this article as work performed under the auspices of the U.S. Department of Energy. Los Alamos National Laboratory strongly supports academic freedom and a researcher's right to publish; as an institution, however, the Laboratory does not endorse the viewpoint of a publication or guarantee its technical correctness. 


\section{DISCLAIMER}

This report was prepared as an account of work sponsored by an agency of the United States Government. Neither the United States Government nor any agency thereof, nor any of their employees, make any warranty, express or implied, or assumes any legal liability or responsibility for the accuracy, completeness, or usefulness of any information, apparatus, product, or process disclosed, or represents that its use would not infringe privately owned rights. Reference herein to any specific commercial product, process, or service by trade name, trademark, manufacturer, or otherwise does not necessarily constitute or imply its endorsement, recommendation, or favoring by the United States Government or any agency thereof. The views and opinions of authors expressed herein do not necessarily state or reflect those of the United States Government or any agency thereof. 


\section{DISCLAIMER}

Portions of this document may be illegible in electronic image products. Images are produced from the best available original document. 


\section{Imaging Time-of-Flight Ion Mass Spectrograph}

Herbert O. Funsten and David J. McComas

\begin{abstract}
This is the final report of a three-year, Laboratory-Directed Research and Development (LDRD) project at the Los Alamos National Laboratory (LANL). The primary objective of this project was to develop and demonstrate a new type of time-of-flight mass spectrometer having a duty cycle of unity, which is a factor of $10^{3}-10^{4}$ better than conventional time-offlight spectrometers. The spectrometer concept was demonstrated, and an additional pre-filtration technique was developed and demonstrated. The two techniques are patents pending.
\end{abstract}

PECENER

DEC 132080

OSTI

\section{Background and Research Objectives:}

Since J.J. Thomson's first mass spectrometer results in 1912[1] mass spectrometry has developed into a powerful tool to probe the composition of matter. Time-of-flight mass spectrometry (TOFMS) techniques were refined in the 1960's, but the method was supplanted by high-mass-resolution magnetic and electric quadrupole methods due to the limited time-resolving capabilities of that decade. However, magnetic and quadrupole methods have certain drawbacks, including the large size, serial (incremental) mass measurement, and difficulty of measuring large molecules that, for example, are used in the biosciences.

These drawbacks are overcome in TOFMS [2], in which an ion's mass is obtained by measuring the time $t$ that an ion of known energy $E$ travels a known distance $d$, i.e. $\mathrm{m}=$ $2 E t^{2} / d^{2}$. In a conventional, gated TOFMS, one electrostatically gates the beam so that a batch of monoenergetic ions enters the drift tube of distance $d$ at time $t_{1}$; an ion in the batch is detected at the end of the drift tube at time $t_{2}$, which depends on the ion velocity. However, a large limitation of conventional TOFMS is an extremely low duty cycle $(<0.001)$ since the system cannot be gated again until ions from the previous gate have traversed the drift tube. Additionally, thin-foil techniques [e.g., McComas and Nordholt, Rev. Sci. Instrum. 61 (1990) 3095] used to generate a start signal require substantially higher ion beam energies, especially for heavy ions, and are typically large in size.

The Imaging Time-of-Flight Ion Mass Spectrograph (ITOFS) that was developed under this project utilizes a continuous beam of ions that are deflected at the entrance of the drift tube to a specific location on the 2-D imaging detector at the other end of the drift tube.

*Principal Investigartor, e-mail: hfunsten@lanl.gov 
The deflection is time-varying, so that the position at which an ion is detected correlates directly with the "start" time at which the ion entered the drift region (or, more specifically, with the time at which the deflection plates were set to the voltage that would send the ion to the detected position). Of course, the time that the ion hits the detector is the "stop" time. The time-of-flight across the drift tube is therefore obtained and, therefore, so is the ion mass. Consequently, this ungated technique results in a duty cycle of unity (the beam entering the drift region is continuous).

A second technology developed as part of this project is a refocusing "Chicane" mass filter that can select a mass range to enter the time-of-flight region. This allows only ion masses within the selected range to enter the TOF section. For example, the mass of base molecules in most chemical weapons resides in a mass range of 200-500 amu. For an air sample input into the mass spectrometer, the appropriate setting of the Chicane filter removes the dominant species from air (e.g., $\mathrm{N}_{2}, \mathrm{Ar}$ ) and only allows masses in the range of 200-500 to enter the TOF section. This greatly enhances both the sensitivity, accuracy, and speed at which a measurement is obtained.

\section{Importance to LANL's Science and Technology Base and National R\&D Needs}

Both ITOFS and the Chicane mass filter allow faster and more accurate measurements than conventional time-of-flight mass spectrometry. As such, these technologies cover a broad spectrum of applications requiring rapid, sensitive measurements of trace species using mass spectrometry. In particular, these technologies directly address Nonproliferation and Counterproliferation and Environmental Stewardship.

\section{Scientific Approach and Accomplishments}

The ITOFS and Chicane mass filter were both fabricated and successfully tested. The following are descriptions of each of these technologies.

Following Fig. 1, an ion's time-of-flight in ITOFS is obtained as follows. First, the ion beam is rastered similarly to a cathode ray tube (CRT) by applying time-varying voltages $V_{H}(t)$ and $V_{V}(t)$ to the raster plates. For example, the scanning system "writes" the first ion beam "line" starting in the upper left corner of the detector and ending at the upper right corner. Upon completion of this line, the next line is written below it at a spacing that allows complete separation of the lines. This is continued until a complete "frame" is written, and the cycle repeats. 
An ion is detected at time $t_{2}$ at a position $(x, y)$ on the position sensitive detector. This position $(x, y)$ corresponds to unique voltages $\mathrm{V}_{\mathrm{H}}\left(\mathrm{t}_{1}\right)$ and $\mathrm{V}_{\mathrm{V}}\left(\mathrm{t}_{1}\right)$ on the horizontal and vertical deflection plates, through which the ion passed at time $t_{l}$. Therefore, ITOFS requires the following information to measure ion mass: ion energy, $t_{2}$, the detected position $(x, y)$, knowledge of the scanning system synchronization to derive $t_{1}$ from $(x, y)$, and the drift length $d$ of the ion. Figure 2 shows results obtained from the prototype using $\mathrm{He}^{+}$using a circular raster.

Several unique features of ITOFS provide important advances in measurement capabilities compared to conventional TOFMS. First, the duty cycle of ITOFS is approximately unity since the start time is uniquely determined by the detected position so that gating of the ion beam is not required. This enables rapid, accurate measurements that cannot be achieved in conventional TOFMS. Second, although the pathlength of ions through the drift section can vary substantially (for example, for trajectories off the central axis of ITOFS), knowledge of the drift distance $d$ of an ion is accurately determined using the ion's detected position $(x, y)$. This enables the drift tube to be shortened without decreasing the error associated with $d$. The ITOFS technology developed under this LDRD project is patent pending.

In contrast to magnetic sector and quadrupole mass spectrometers that typically measure and detect one or a few ion masses at a time, a time-of-flight mass spectrometer such as ITOFS typically measures a wide range of particle masses simultaneously. While this feature enables rapid acquisition of mass spectra, high sensitivity measurement of trace mass species is difficult due to (1) count rate limitations of the detector system, resulting in a majority of counts from abundant mass species and few counts from trace species, and (2) significant background from abundant mass species that can mask or produce large errors in trace species analysis. Gated TOFMS sometimes utilize a second gate near the end of the drift section to remove unwanted species from the measurement; however, such gating requires sophisticated, precision electronics for timing of the pulse and duration of the pulse, and the gated scheme has a low duty cycle.

As an example, measurement of chemical and biological warfare (CBW) agents in a sample of air requires an extremely rapid detection and analysis in order to respond to the threat, and proper analysis requires detection of a variety of CBW agents and their dissociation (cracking) byproducts from the ion source. In an air sample having trace amounts of a CBW agent, a mass spectrum derived using TOFMS will contain mostly counts from basic atmospheric constituents in the mass range 1-50 amu (e.g., $\mathrm{N}_{2}, \mathrm{H}_{2} \mathrm{O}$, and $\mathrm{Ar}$ ), whereas the targeted $\mathrm{CBW}$ agent lies within the mass range of 50-400 amu and will produce few counts. 
This measurement either provides rapid, low accuracy results or delayed, high accuracy results. The presence of major atmospheric constituents in the measurement (1) decreases the count rate of targeted species due to count rate limitations of the detector, and (2) introduces a noise background that can interfere with the measurement of the trace species.

We have developed an adjustable mass range filter, in which a user can select the low end and high end of a mass range that will be admitted into the drift section of a timeof-flight mass spectrometer. Therefore, when looking for airborne chemical or biological warfare agents, the sample can be directly input into the ion source, and the filter an be adjusted to reject air species (masses 1-50) and accept heavier ions characteristic of the agents of interest.

The Chicane mass filter consists of 4 dipole magnets with polarities as shown in Figs. 3, and ions traversing the magnets spatially disperse and refocus according to mass. The chicane magnets are placed in a symmetric geometry so that ions that reach the halfway point have trajectories that are parallel to the incident beam, and ions at the exit follow a trajectory that is collinear with the incident beam. At the point of maximum separation (the halfway point), adjustable blocking plates are used to reject ions heavier and lighter than the targeted mass range. Ions within the targeted mass range are magnetically refocused back into a beam that is collinear with the incident beam and are subsequently input into the timeof-flight mass spectrometer. The unique features of this apparatus include (1) mass range selectivity in which the selected masses exit the apparatus in a collinear beam and (2) simple and independent control of the lower and upper masses of the mass range that exits the apparatus. The Chicane, shown in Fig. 4, has been successfully tested and the concept is patent pending.

\section{Publications/Patents}

Funsten, H.O., and D.J. McComas, Time-of-Flight Ion Mass Spectrograph, U.S. Patent Pending, 1999.

H.O. Funsten, Chicane Mass Filter, US Patent Pending, 1999.

\section{References}

[1] Thomson, J.J., Philos. Mag., 24209 (1912).

[2] Guilhaus, M., J. Mass Spectrom., 30 , 1519, (1995). 


\section{Enter any figure captions here}

Figure 1: The schematic shows the basic operation of the time-of-flight mass spectrograph. An ion is deflected by raster plates with voltages $V_{\mathrm{V}}\left(t_{1}\right)$ and $V_{\mathrm{H}}\left(t_{1}\right)$ when it enters the TOF tube at time $t_{1}$. It is eventually detected at time $t_{2}$ at the position $(x, y)$. The detected position $(x, y)$ provides unique information about the voltages $V_{\mathrm{v}}\left(t_{1}\right)$ and $V_{\mathrm{H}}\left(t_{1}\right)$ at the time $t_{1}$ that the ion passed the raster plates, so $t_{1}$ can be uniquely determined. Also, the distance of travel $d$ of the ion is accurately measured using $(x, y)$. Ion speed is obtained using $d /\left(t_{2}-t_{1}\right)$.

Figure 2: A time-of-flight spectrum of a ${ }^{4} \mathrm{He}$ beam was derived using the prototype apparatus.

Figure 3: A schematic of the Chicane mass filter illustrating the selection of a mass range from 50-400 amu to pass through the filter to the TOF mass spectrometer.

Figure 4: The prototype of the Chicane mass filter uses off-the-shelf magnets and iron pole pieces. The exit horizontal slit is wide to allow for detection and analysis of trajectories that do not exit the filter collinear with the entrance beam. The hole on the long side of the filter holds the adjustable aperture. 


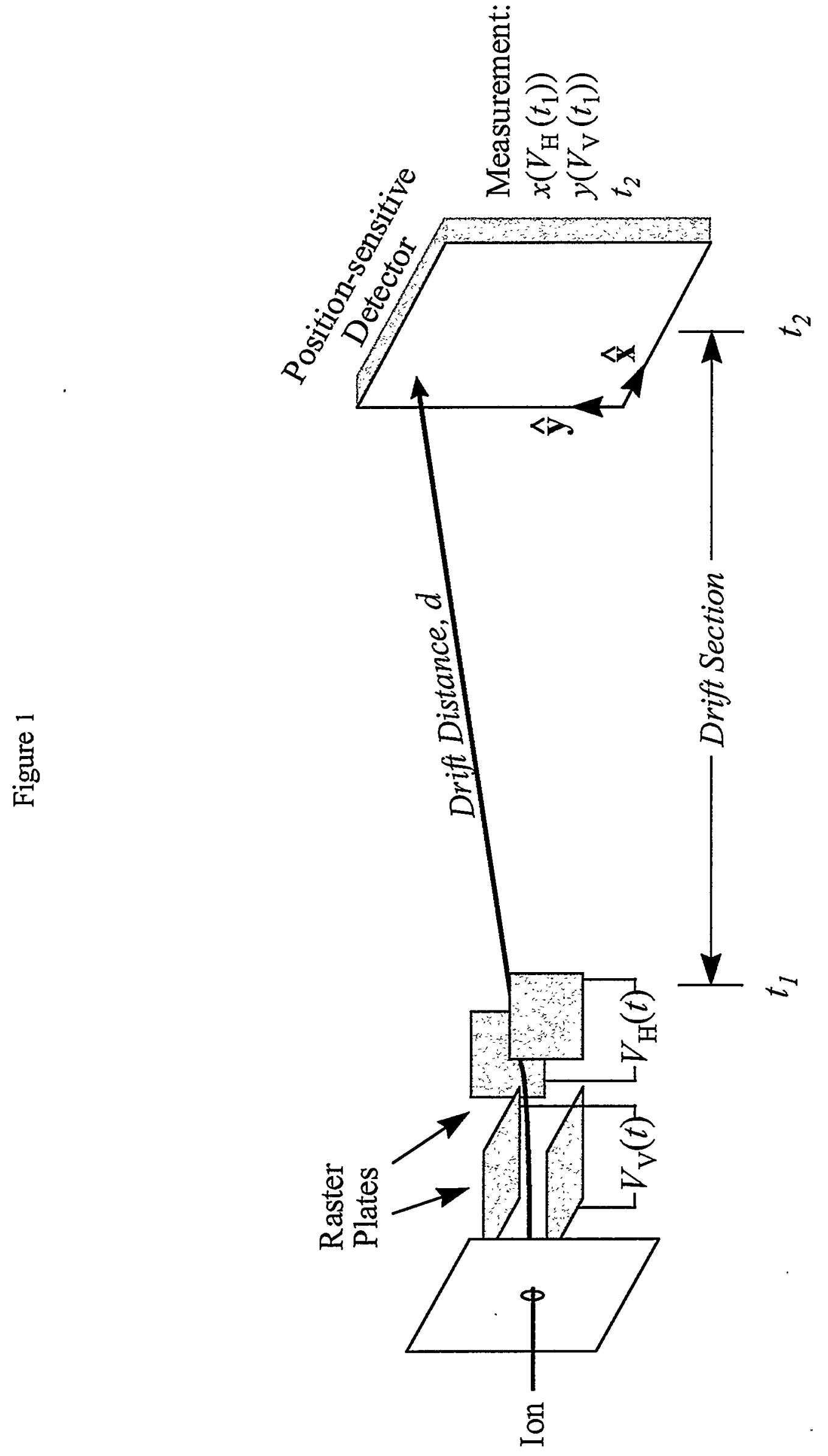









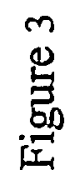

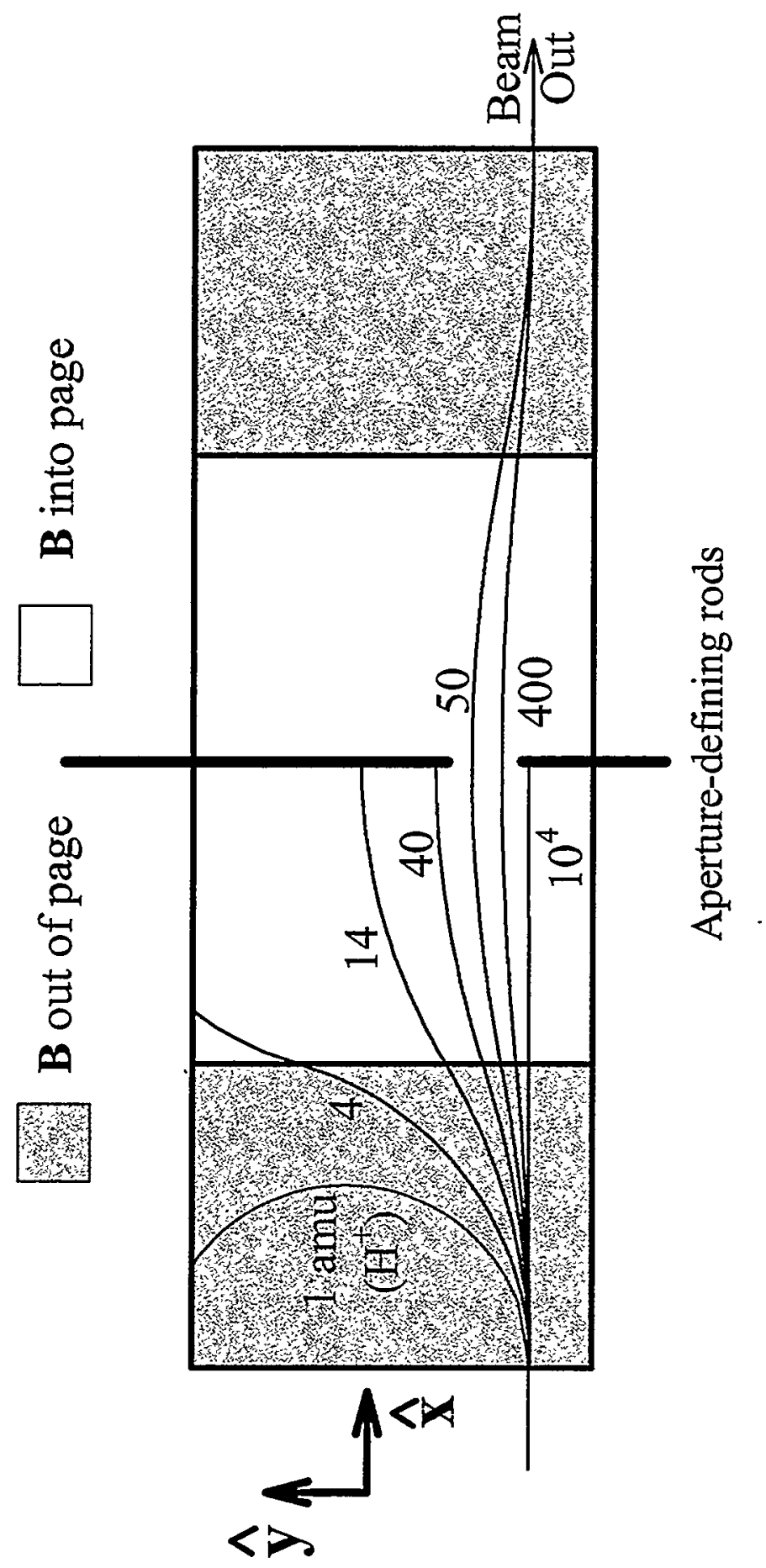




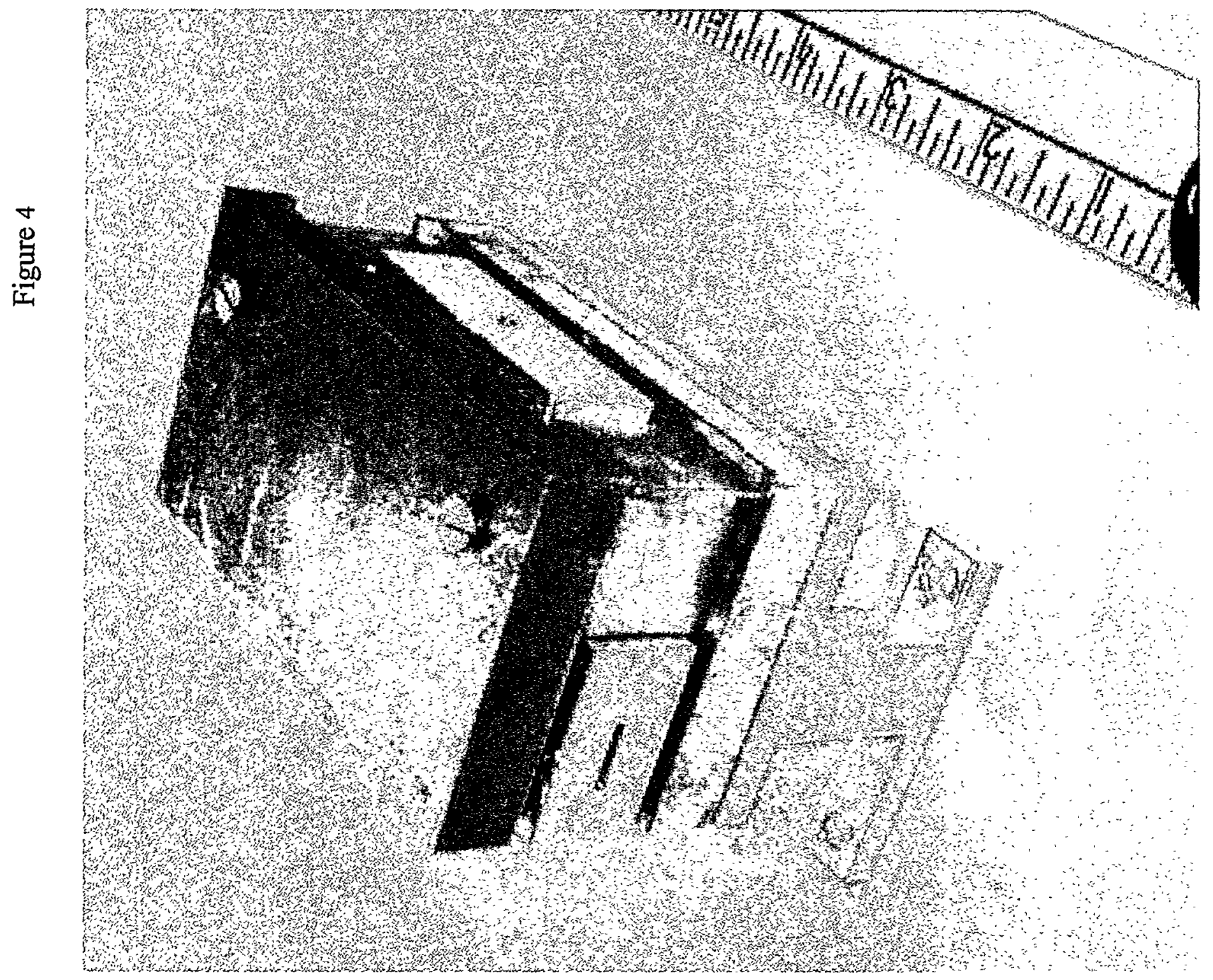

